\title{
Non-Motor Symptoms in Treated and Untreated Chinese Patients with Early Parkinson's Disease
}

\author{
Hui Zhang, ${ }^{1}$ Zhuqin Gu, ${ }^{2}$ Jing An, ${ }^{1}$ Chaodong Wang ${ }^{1}$ and Piu Chan ${ }^{1,2,3,4}$ \\ ${ }^{1}$ Department of Neurobiology and Neurology, Beijing Institute of Geriatrics, Xuanwu Hospital of Capital Medical \\ University, Beijing, P.R. China \\ ${ }^{2}$ Chinese National Human Genome Center, Beijing, P.R. China \\ ${ }^{3}$ Key Laboratory of Ministry of Education on Neurodegenerative Disorder, Beijing, P.R. China \\ ${ }^{4}$ Beijing Key Laboratory on Parkinson's Disease, Beijing, P.R. China
}

Non-motor symptoms (NMS) are important preclinical features of Parkinson's disease (PD) and have become the leading cause of poor quality of life with disease progression. There are little data on how antiparkinsonian medications influence the NMS in PD at early stage. In this study, we explored the distribution of NMS in treated and untreated PD and investigated the association between NMS and antiparkinsonian medications in Chinese patients with early PD. Subjects were enrolled from a Chinese PD patient cohort based on 2 clinical trials. Face-to-face interviews and evaluations were performed for clinical information. NMS were compared in patients with or without antiparkinsonian treatment, and between subgroups of dopaminergic medications. Eight hundred and sixteen PD patients were enrolled in this study, of whom 428 were newly diagnosed PD. Only 5 in 646 patients who completed all these NMS measurements $(0.6 \%)$ were free of NMS. PD patients with antiparkinsonian medications had a significantly higher frequency of poor sleep $(p=0.001)$, depression $(p=0.0001)$ and constipation $(p=0.0001)$ after adjusted gender, onset age, duration, and Hoehn \& Yahr stage. Moreover, patients treated by levodopa plus dopamine agonist had a higher percentage of bad sleepers (adjusted $p=0.040$ ), and correlation analysis revealed that Levodopa Equivalent Dose (LED) was associated with constipation (coefficient $0.146, p=0.005)$. These findings suggest that although NMS exist in the prodromal stage of PD, antiparkinsonian treatment is associated with increased frequency of some NMS, which may challenge the management for PD.

Keywords: antiparkinsonian medication; Chinese patients; dopaminergic treatment; non-motor symptoms;

Parkinson's disease

Tohoku J. Exp. Med., 2014 February, 232 (2), 129-136. C 2014 Tohoku University Medical Press

\section{Introduction}

Parkinson's disease (PD) is characterized by motor symptoms including resting tremor, rigidity, bradykinesia and postural instability. However, less is known about nonmotor symptoms (NMS), which are very common and often go under-recognized and unreported by both patients and clinicians. NMS are comprised of a large range of symptoms including neuropsychiatric, autonomic, sleep, sensory, gastrointestinal, and other symptoms (Chaudhuri and Schapira 2009). They are increasingly prevalent over the course of the illness and have become important determinants of patients' quality of life with disease progression in PD (Duncan et al. 2013). NMS could occur at any stage of $\mathrm{PD}$, even preceding the onset of motor symptoms, which is correlated with the Braak stages of Lewy pathology in PD (Braak et al. 2003; Wolters and Braak 2006).
Although antiparkinsonian medication is important for improving the motor performance of PD, its effect on NMS remains a matter of debate. Recently, a study with positron emission tomography highlighted the dopaminergic dysfunction in the hypothalamus that might contribute to the development of several NMS in PD, such as sleep disturbances and autonomic dysfunction, which provided the in vivo evidence of potential benefits from dopaminergic treatment for NMS management (Politis et al. 2008). Several NMS might be responsive to dopaminergic treatments, such as depression and constipation, whereas other NMS might be exacerbated with antiparkinsonian drugs, such as hallucinations and excessive daytime sleepiness (Chaudhuri and Schapira 2009). However, all the data were from clinical trials with a specific drug, and little is known about dopaminergic treatments on NMS in epidemiological studies. Here, we present a cross-sectional study to describe the dis-

Received December 30, 2013; revised and accepted February 3, 2014. Published online February 25, 2014; doi: 10.1620/tjem.232.129.

Correspondence: Piu Chan, Ph.D., Department of Neurology and Neurobiology, Xuanwu Hospital of Capital Medical University, \#45

Changchun Street, Beijing 100053, P.R. China.

e-mail: pbchan_xw@hotmail.com or pbchan90@gmail.com 
tributions of NMS in Chinese PD patients with or without antiparkinsonian treatment and to investigate the effects of medications on NMS.

\section{Materials and Methods}

Subjects

Subjects were recruited from two trial-based multicenter PD cohorts, established by the Chinese Parkinson Study Group (CPSG) from 2005-2008. CPSG consists of 42 clinical centers and is coordinated by Xuanwu Hospital of Capital Medical University in Beijing, China. All the clinical centers belong to tertiary hospitals and have at least one movement disorders specialist. Individuals with PD were recruited from their movement disorders clinic. PD patients were diagnosed using the UK Brain Bank criteria (Litvan et al. 2003). Primary medical records were reviewed by specialists to confirm the diagnosis. Atypical and secondary parkinsonism were excluded. Patients with cognitive impairment (Mini-Mental Status Examination, MMSE $\leq 24$ ) were also excluded due to the inability to complete the scales. Cases with onset age $\geq 30$ years, Hoehn \& Yahr (H \& Y) stage $\leq 3.0$, and with completed baseline information were enrolled in our study.

\section{Data collection}

Demographic information and clinical characteristics were collected by face-to-face interviews. Motor symptoms of PD were evaluated with the Unified Parkinson Disease Rating Scale (UPDRS). NMS of PD were evaluated using specific questionnaires. Depression was measured by the full-length, 20-item version of the Center for Epidemiological Studies Depression Scales (CES-D). The Pittsburg Sleep Quality Index (PSQI) was used to evaluate the quality of sleep. Subscales of memory, orientation, and identification in the Alzheimer's Disease Assessment Scale-Cognitive (ADAS-Cog) were used to measure cognition status. Constipation was assessed according to item 21 on the Non-Motor Symptoms Scale (NMSS), by which constipation was defined as one bowel movement every other day or less. Fatigue was determined by a yes-or-no question.

\section{Medication}

Detailed information of antiparkinsonian medications in their lives was collected. Patients who had/were having antiparkinsonian medications were defined as 'treated' ones. Prescription details of Levodopa, Levodopa-Carbidopa controlled-release (Sinemet), Piribedil, Entacapone, Trihexyphenidyl (Artane), Selegiline and Amantadine were recorded.

\section{Ethics}

This study was approved by the Ethics Committee of XuanWu Hospital of Capital Medical University. The use of human subjects was carried out with adequate understanding and written consent of the subjects or their legal guardians.

\section{Statistical Analysis}

SPSS11.5 was used for database and analyses. Data for each NMS was only analyzed in the available cases. Subscales of UPDRS III were used to measure motor symptoms. Depression was defined by a CES-D score $\geq 16$. A PSQI score $\leq 5$ was considered to be good sleep quality. Cognition status was measured by the ADAS-Cog total score and also by the subscales of memory, orientation, and identification. Differences of NMS distributions were compared between untreated and treated patients, and also between different medication subgroups. Levodopa Equivalent Dose (LED) was calculated as follows: (regular levodopa dose $\times 1)+($ levodopa controlled-release dose $\times 0.75)+($ Piribedil dose $\times 1)+(($ regular levodopa dose $\times 1+$ levodopa controlled-release dose $\times 0.75) \times 0.33)$ if taking entacapone (Tomlinson et al. 2010). The chi-square test was performed to analyze the differences in discrete data, and analysis of variance (ANOVA) test was used for continuous data. Logistic regression model and linear model were used to control the effects of gender, onset age, disease duration, and $\mathrm{H} \& \mathrm{Y}$ stage. Pearson correlation coefficient and regression models were used to analyze the associations for LED and NMS. $P<0.05$ was considered to be statistically significant.

\section{Results}

\section{Demographic and clinical characteristics}

A total of 816 subjects were enrolled in our study, of which 428 cases were non-treated PD, and 388 were treated with antiparkinsonian medications. All the PD patients who had received antiparkinsonian therapy had dopaminergic drugs. Patients under treatment were much older than those untreated (mean age $64.29 \pm 9.57$ vs. $60.62 \pm 10.77$ years, $p$ $=0.0001)$, while there was no difference in gender distribution in two groups $(p=0.222)$. The basic clinical features of PD are compared in Table 1. Treated patients had a longer disease duration $(41.80 \pm 22.92$ vs. $22.97 \pm 21.00$ months) and more severe disease stages (H \& Y stage 1.98 \pm 0.66 vs. $1.61 \pm 0.57$ ) and motor symptoms (UPDRS-III score $23.47 \pm 11.47$ vs. $16.78 \pm 9.59)$ than untreated ones. The average LED was $439.09 \pm 213.10 \mathrm{mg} /$ day in treated patients, and 20.9\% (81/388), $14.7 \%$ (57/388), 34.0\% $(132 / 388)$ of the treated patients were also taking Trihexyphenidyl, Selegiline and Amantadine, respectively.

\section{NMS distribution in treated and untreated $P D$}

PSQI, CES-D, constipation, fatigue, and ADAS-Cog measurements were available in 668, 814, 682, 659 and 813 cases, respectively. A total of 646 patients completed all these NMS measurements, and had a longer disease duration than those who did not complete all the measurements (33.66 \pm 24.82 vs. $25.31 \pm 18.37$ months, $p=0.0001)$ but had similar age at evaluation, age at onset, H \& Y stage, and gender distribution. In those who had all the NMS data, only five patients $(0.6 \%)$ reported no NMS, while 117 patients (18.1\%) had all the five symptoms. Among all the cases, $48(7.4 \%), 111(17.2 \%), 174(26.9 \%)$, and 191 (29.6\%) cases had one, two, three and four NMS, respectively.

The distribution of NMS is listed in Table 2. Sleep quality assessed by PSQI suggested that $68.6 \%$ of the patients were bad sleepers. The percentage was even higher in patients treated with antiparkinsonian medications (75.5\%) as compared to those untreated $(59.2 \%)$ ( $p=$ 0.0001 ). Totally $42.6 \%$ PD patients were suffering from depression. $54.4 \%$ treated patients presented depression, which were significantly more frequent than observed in 
Table 1. Demographic and clinical characteristics of the enrolled PD patients.

\begin{tabular}{|c|c|c|c|c|c|}
\hline \multicolumn{2}{|c|}{ Variables } & \multirow{2}{*}{$\begin{array}{c}\text { Total } \\
508(62.3)\end{array}$} & \multirow{2}{*}{$\begin{array}{c}\text { Untreated PD } \\
258(60.3)\end{array}$} & \multirow{2}{*}{$\begin{array}{l}\text { Treated PD } \\
250(64.4)\end{array}$} & \multirow{2}{*}{$\begin{array}{l}P \text { value } \\
0.222\end{array}$} \\
\hline Gender & Male & & & & \\
\hline & Female & $308(37.7)$ & $170(39.7)$ & $138(35.6)$ & \\
\hline Age (ye & & $62.36 \pm 10.37$ & $60.62 \pm 10.77$ & $64.29 \pm 9.57$ & 0.0001 \\
\hline \multicolumn{2}{|c|}{ Onset age (years) } & $59.78 \pm 10.19$ & $58.75 \pm 10.55$ & $60.92 \pm 9.67$ & 0.002 \\
\hline \multicolumn{2}{|c|}{ Duration (months) } & $31.92 \pm 23.86$ & $22.97 \pm 21.00$ & $41.80 \pm 22.92$ & 0.0001 \\
\hline \multicolumn{2}{|l|}{$H \& Y$} & $1.78 \pm 0.64$ & $1.61 \pm 0.57$ & $1.98 \pm 0.66$ & 0.0001 \\
\hline \multicolumn{2}{|c|}{ UPDRS total score } & $30.05 \pm 15.15$ & $25.11 \pm 12.98$ & $35.51 \pm 15.51$ & 0.0001 \\
\hline \multicolumn{2}{|c|}{ UPDRSIII subscore } & $19.96 \pm 11.04$ & $16.78 \pm 9.59$ & $23.47 \pm 11.47$ & 0.0001 \\
\hline \multicolumn{3}{|c|}{$\operatorname{LED}(N=376 / 388, \mathrm{mg} /$ day $)$} & NA & $439.09 \pm 213.10$ & \\
\hline \multicolumn{3}{|c|}{ Trihexyphenidyl $(N=81 / 388, \mathrm{mg}$ /day $)$} & NA & $4.10 \pm 2.03$ & \\
\hline \multicolumn{3}{|c|}{ Selegiline $(N=57 / 388, \mathrm{mg} /$ day $)$} & NA & $5.96 \pm 2.53$ & \\
\hline \multicolumn{3}{|c|}{ Amantadine $(N=132 / 388, \mathrm{mg} /$ day $)$} & NA & $210.23 \pm 51.59$ & \\
\hline
\end{tabular}

PD, Parkinson's disease; H \& Y, Hoehn \& Yahr stage; UPDRS, Unified Parkinson Disease Rating Scale; LED, Levodopa Equivalent Dose; NA, not applicable.

Continuous variables were presented as mean \pm S.D.; discrete variables were presented as numbers (\%).

Table 2. Associations between NMS and antiparkinsonian treatment.

\begin{tabular}{|c|c|c|c|c|c|c|}
\hline \multicolumn{2}{|c|}{ Variables } & \multirow{2}{*}{$\begin{array}{c}\text { Total } \\
210(31.4)\end{array}$} & \multirow{2}{*}{$\begin{array}{c}\text { Untreated PD } \\
116(40.8)\end{array}$} & \multirow{2}{*}{$\begin{array}{l}\text { Treated PD } \\
94(24.5)\end{array}$} & \multirow{2}{*}{$\begin{array}{l}P \text { value } \\
0.0001\end{array}$} & \multirow{2}{*}{$\begin{array}{c}\begin{array}{c}\text { Adjusted } \\
P \text { value* }\end{array} \\
0.001\end{array}$} \\
\hline Sleep quality & Good sleeper & & & & & \\
\hline & Bad sleeper & $458(68.6)$ & $168(59.2)$ & $290(75.5)$ & & \\
\hline \multirow[t]{2}{*}{ Depression } & CES-D score $<16$ & $467(57.4)$ & $291(68.0)$ & $176(45.6)$ & 0.0001 & 0.0001 \\
\hline & CES-D score $\geq 16$ & $347(42.6)$ & $137(32.0)$ & $210(54.4)$ & & \\
\hline \multirow[t]{2}{*}{ Constipation } & No & $335(49.1)$ & $194(65.8)$ & $141(36.4)$ & 0.0001 & 0.0001 \\
\hline & Yes & $347(50.9)$ & $101(34.2)$ & $246(63.6)$ & & \\
\hline \multirow[t]{2}{*}{ Fatigue } & No & $208(31.6)$ & $100(34.1)$ & $108(29.5)$ & 0.205 & 0.871 \\
\hline & Yes & $451(68.4)$ & $193(65.9)$ & $258(70.5)$ & & \\
\hline ADAS-Cog & $(N=813)$ & $5.54 \pm 3.30$ & $5.43 \pm 3.64$ & $5.66 \pm 2.88$ & 0.320 & 0.195 \\
\hline Memory & & $3.52 \pm 1.66$ & $3.35 \pm 1.71$ & $3.72 \pm 1.59$ & 0.002 & 0.148 \\
\hline Orientation & & $0.14 \pm 0.59$ & $0.16 \pm 0.60$ & $0.11 \pm 0.57$ & 0.205 & 0.001 \\
\hline Identification & & $1.60 \pm 1.85$ & $1.63 \pm 2.10$ & $1.56 \pm 1.52$ & 0.548 & 0.047 \\
\hline
\end{tabular}

PD, Parkinson's disease; NMS, non-motor symptoms; CES-D, Center for Epidemiological Studies Depression Scales;

ADAS-Cog, Alzheimer's Disease Assessment Scale-Cognitive.

Continuous variables were presented as mean \pm S.D.; discrete variables were presented as numbers (\%).

*Adjusted by gender, onset age, disease duration, and Hoehn \& Yahr stage.

non-treated patients $(32.0 \%, p=0.0001)$. The overall frequency of constipation was $50.9 \%$ in PD patients, among whom $63.6 \%$ were treated. Fatigue was reported in $68.4 \%$ patients, which was distributed in the treated and nontreated groups with a similar frequency $(70.5 \%$ vs. $65.9 \%$, $p=0.205)$. The average subscore was 0.14 for orientation and 1.60 for identification. The average subscore for memory was 3.52 , which was significantly higher in the treated group than in the untreated group $(p=0.002)$. In the logistic regression analysis adjusted for gender, onset age, duration and $\mathrm{H} \& \mathrm{Y}$ stage, antiparkinsonian treatment was associated with bad sleep quality $(p=0.001)$, depression $(p=$ $0.0001)$, constipation $(p=0.0001)$, orientation $(p=0.001)$ and identification $(p=0.047)$, while memory, which appeared to be associated with medication, was not significantly different in the two groups after the regression models were performed (memory score $3.35 \pm 1.71$ vs. $3.72 \pm$ $1.59, p=0.148)$.

\section{NMS in PD patients on dopaminergic treatments}

To confirm the association between treatment and NMS, we further compared the occurrence of NMS between specific dopaminergic medication groups. Eight patients did not take medications at time of evaluation, and four more patients did not take the medications included in LED equation; thus, these patients were excluded in the analysis of LED and NMS. For the remained 376 treated patients, 305 (81.1\%) patients were on levodopa therapy, 3 
Table 3. Differences of disease characteristics and NMS between the PD patients taken levodopa and levodopa plus dopamine agonist.

\begin{tabular}{|c|c|c|c|c|c|}
\hline \multicolumn{2}{|c|}{ Variables } & \multirow{2}{*}{$\begin{array}{c}\text { Levodopa } \\
(n=305)\end{array}$} & \multirow{2}{*}{$\begin{array}{c}\text { Levodopa+Dopamine } \\
\text { Agonist }(n=68) \\
50(73.5)\end{array}$} & \multirow{2}{*}{$\begin{array}{c}P \text { value } \\
0.089\end{array}$} & \multirow[t]{2}{*}{$\begin{array}{l}\text { Adjusted } \\
P \text { value* }\end{array}$} \\
\hline Gender & Male & & & & \\
\hline & Female & $114(37.4)$ & $18(26.5)$ & & \\
\hline Age (years) & & $64.65 \pm 9.37$ & $62.31 \pm 10.58$ & 0.070 & \\
\hline Onset age (years) & & $61.35 \pm 9.53$ & $58.69 \pm 10.31$ & 0.041 & \\
\hline Duration (months) & & $41.48 \pm 23.58$ & $43.16 \pm 19.91$ & 0.585 & \\
\hline$H \& Y$ & & $1.98 \pm 0.67$ & $1.96 \pm 0.59$ & 0.833 & \\
\hline UPDRS total score & & $35.68 \pm 15.62$ & $34.43 \pm 14.87$ & 0.548 & \\
\hline UPDRSIII subscore & & $23.50 \pm 11.73$ & $23.20 \pm 10.29$ & 0.845 & \\
\hline LED (mg/day) & & $429.08 \pm 220.82$ & $499.69 \pm 151.57$ & 0.013 & \\
\hline Trihexyphenidyl & & $70(23.0)$ & $9(13.2)$ & 0.076 & \\
\hline Selegiline & & $48(15.7)$ & $8(11.8)$ & 0.407 & \\
\hline Amantadine & & $108(35.4)$ & $19(27.9)$ & 0.240 & \\
\hline \multirow[t]{2}{*}{ Sleep quality } & Good sleeper & $80(26.5)$ & $12(17.6)$ & \multirow{2}{*}{0.127} & \multirow{2}{*}{0.040} \\
\hline & Bad sleeper & $222(73.5)$ & $56(82.4)$ & & \\
\hline \multirow[t]{2}{*}{ Depression } & CES-D score $<16$ & $129(42.6)$ & $37(54.4)$ & \multirow{2}{*}{0.076} & \multirow{2}{*}{0.120} \\
\hline & CES-D score $\geq 16$ & $174(57.4)$ & $31(45.6)$ & & \\
\hline \multirow[t]{2}{*}{ Constipation } & No & $112(36.8)$ & $23(33.8)$ & \multirow{2}{*}{0.482} & \multirow{2}{*}{0.409} \\
\hline & Yes & $192(63.2)$ & $45(66.2)$ & & \\
\hline \multirow[t]{2}{*}{ Fatigue } & No & $76(26.4)$ & $26(40.6)$ & \multirow{2}{*}{0.023} & \multirow{2}{*}{0.060} \\
\hline & Yes & $212(73.6)$ & $38(59.4)$ & & \\
\hline ADAS-Cog score & $(N=373)$ & $5.60 \pm 2.89$ & $6.01 \pm 3.00$ & 0.295 & 0.142 \\
\hline Memory & & $3.71 \pm 1.61$ & $3.80 \pm 1.49$ & 0.688 & 0.447 \\
\hline Orientation & & $0.08 \pm 0.37$ & $0.25 \pm 1.10$ & 0.031 & 0.066 \\
\hline Identification & & $1.55 \pm 1.53$ & $1.63 \pm 1.56$ & 0.698 & 0.407 \\
\hline
\end{tabular}

H \& Y, Hoehn \& Yahr stage; UPDRS, Unified Parkinson Disease Rating Scale; LED, Levodopa Equivalent Dose; CES-D, Center for Epidemiological Studies Depression Scales; ADAS-Cog, Alzheimer's Disease Assessment Scale-Cognitive.

Continuous variables were presented as mean \pm S.D.; discrete variables were presented as numbers (\%).

*Adjusted by gender, onset age, disease duration, and Hoehn \& Yahr stage.

(0.8\%) patients on dopamine agonist therapy, 68 (18.1\%) patients on levodopa plus dopamine agonist therapy. Therefore, we compared NMS distribution in levodopa and levodopa plus agonists treated groups (Table 3). Patients taking levodopa plus agonists had a younger onset age $(58.69 \pm 10.31$ vs. $61.35 \pm 9.53$ years, $p=0.041)$, and higher LED (499.69 \pm 151.57 vs. $429.08 \pm 220.82 \mathrm{mg} /$ day, $p=0.013$ ). While other disease characteristics did not differ significantly between the two groups. Percentages of patients using Trihexyphenidyl, Selegiline and Amantadine were not statistically different between the two groups, though the percentages in levodopa group were slighly higher $(23.0 \%$ vs. $13.2 \%$ for Trihexyphenidyl; $15.7 \%$ vs. $11.85 \%$ for Selegiline; $35.4 \%$ vs. $27.9 \%$ for Amantadine). Patients with levodopa plus agonists treatments were more likely bad sleepers $(82.4 \%$ vs. $73.5 \%, p=0.127)$, less likely to have fatigue $(59.4 \%$ vs. $73.6 \%, p=0.023)$ and have lower score for orientation $(0.25 \pm 1.10$ vs. $0.08 \pm 0.37, p=$ 0.031). But constipation was not different between the groups $(66.2 \%$ vs. $63.2 \%, p=0.482)$. However, when adjusted by potential confounding factors, only sleep qual- ity remained statistically significant (adjusted $p=0.040$ ).

We further analyzed the association between LED and NMS in treated patients (Table 4). It appeared that LED was associated only with constipation (coefficient $0.146, p$ $=0.005)$, while sleep quality, depression and fatigue were significantly associated with each other $(p<0.01)$. In addition, constipation was also associated with cognition especially for memory $(p<0.01)$. Furthermore, when we used regression models adjusting potential confounding factors and also LED for the associations among NMS, the associations among sleep, depression and fatigue remained significant $(p<0.02)$ (Table 5).

\section{Discussion}

Our study strengthens the notion that NMS are frequent and affect most of the PD patients. Only $0.6 \%$ subjects were free of NMS, and $18.1 \%$ patients had all five NMS we investigated. More than half of the patients experienced bad sleep quality, fatigue, or constipation, and over $40 \%$ of the patients suffered from depression. Even in untreated patients, the prevalence of NMS was high. This 
Table 4. Pearson correlation coefficient analysis for LED and NMS (only in treated patients).

\begin{tabular}{|c|c|c|c|c|c|c|c|}
\hline & & LED & Sleep & Depression & Constipation & Fatigue & ADAS-Cog \\
\hline \multirow[t]{3}{*}{ LED } & $\mathrm{r}$ & 1 & 0.089 & 0.033 & $0.146^{* *}$ & -0.009 & 0.061 \\
\hline & $P$ value & - & 0.086 & 0.519 & 0.005 & 0.870 & 0.239 \\
\hline & $\mathrm{N}$ & 376 & 373 & 374 & 375 & 355 & 373 \\
\hline \multirow[t]{3}{*}{ Sleep } & $\mathrm{r}$ & 0.089 & 1 & $0.232 * *$ & 0.025 & $0.185^{* *}$ & -0.086 \\
\hline & $P$ value & 0.086 & - & 0.0001 & 0.627 & 0.0001 & 0.093 \\
\hline & $\mathrm{N}$ & 373 & 384 & 383 & 384 & 363 & 382 \\
\hline \multirow[t]{3}{*}{ Depression } & $\mathrm{r}$ & 0.033 & $0.232 * *$ & 1 & 0.062 & $0.229 * *$ & 0.076 \\
\hline & $P$ value & 0.519 & 0.0001 & - & 0.227 & 0.0001 & 0.136 \\
\hline & $\mathrm{N}$ & 374 & 383 & 386 & 386 & 365 & 384 \\
\hline \multirow[t]{3}{*}{ Constipation } & $\mathrm{r}$ & $0.146^{* *}$ & 0.025 & 0.062 & 1 & 0.021 & $0.140 * *$ \\
\hline & $P$ value & 0.005 & 0.627 & 0.227 & - & 0.696 & 0.006 \\
\hline & $\mathrm{N}$ & 375 & 384 & 386 & 387 & 366 & 385 \\
\hline \multirow[t]{3}{*}{ Fatigue } & $\mathrm{r}$ & -0.009 & $0.185^{* *}$ & $0.229 * *$ & 0.021 & 1 & -0.003 \\
\hline & $P$ value & 0.870 & 0.0001 & 0.0001 & 0.696 & - & 0.959 \\
\hline & $\mathrm{N}$ & 355 & 363 & 365 & 366 & 366 & 366 \\
\hline \multirow[t]{3}{*}{ ADAS-Cog } & $\mathrm{r}$ & 0.061 & -0.086 & 0.076 & $0.140 * *$ & -0.003 & 1 \\
\hline & $P$ value & 0.239 & 0.093 & 0.136 & 0.006 & 0.959 & - \\
\hline & $\mathrm{N}$ & 373 & 382 & 384 & 385 & 366 & 385 \\
\hline \multirow[t]{3}{*}{ Memory } & $\mathrm{r}$ & 0.059 & -0.079 & 0.073 & $0.160 * *$ & 0.014 & $0.796^{* *}$ \\
\hline & $P$ value & 0.257 & 0.121 & 0.155 & 0.002 & 0.794 & 0.0001 \\
\hline & $\mathrm{N}$ & 373 & 382 & 384 & 385 & 366 & 385 \\
\hline \multirow[t]{3}{*}{ Orientation } & $\mathrm{r}$ & 0.058 & -0.030 & 0.025 & 0.060 & 0.041 & $0.355^{* *}$ \\
\hline & $P$ value & 0.261 & 0.562 & 0.627 & 0.242 & 0.438 & 0.0001 \\
\hline & $\mathrm{N}$ & 373 & 382 & 384 & 385 & 366 & 385 \\
\hline \multirow[t]{3}{*}{ Identification } & $\mathrm{r}$ & 0.017 & -0.058 & 0.086 & 0.099 & -0.040 & $0.798 * *$ \\
\hline & $P$ value & 0.740 & 0.258 & 0.093 & 0.052 & 0.450 & 0.0001 \\
\hline & $\mathrm{N}$ & 373 & 382 & 384 & 385 & 366 & 385 \\
\hline
\end{tabular}

LED, Levodopa Equivalent Dose; NMS, non-motor symptoms; ADAS-Cog, Alzheimer's Disease Assessment Scale-Cognitive. $\mathrm{r}$ for Pearson correlation coefficient; $\mathrm{N}$ for Number of patients who completed corresponding NMS measurement.

$* * p<0.01$.

Table 5. Regression models for associations among NMS (only in treated patients).

\begin{tabular}{llccccc}
\hline & & Sleep & Depression & Constipation & Fatigue & ADAS-Cog \\
\hline \multirow{2}{*}{ Sleep } & Wald & - & $11.664^{* *}$ & 0.414 & $6.676^{*}$ & $5.985^{*}$ \\
& $P$ value & - & 0.001 & 0.520 & 0.010 & 0.014 \\
\multirow{2}{*}{ Depression } & Wald & $10.941^{* *}$ & - & 0.062 & $6.374^{*}$ & 3.411 \\
& $P$ value & 0.001 & - & 0.803 & 0.012 & 0.065 \\
Constipation & Wald & 0.410 & 0.098 & - & 0.020 & 2.293 \\
& $P$ value & 0.522 & 0.754 & - & 0.888 & 0.130 \\
Fatigue & Wald & $6.511^{*}$ & $6.692^{*}$ & 0.047 & - & 0.275 \\
& $P$ value & 0.011 & 0.010 & 0.828 & - & 0.600 \\
ADAS-Cog & Beta & $-0.126^{*}$ & 0.102 & 0.080 & -0.026 & - \\
& $P$ value & 0.019 & 0.064 & 0.135 & 0.635 & -
\end{tabular}

NMS, non-motor symptoms; ADAS-Cog, Alzheimer's Disease Assessment Scale-Cognitive.

Adjusted by gender, onset age, disease duration, Hoehn \& Yahr stage, LED.

Wald and $P$ value by multiple logistic regression analysis; Beta and $P$ value by linear regression analysis.

$* p<0.05 ; * *<0.01$. 
is consistent with the PRIAMO study (Barone et al. 2009). They evaluated 12 NMS domains in 1,072 PD patients, and found $98.6 \%$ patients reported at least one NMS, and the average number of NMS experienced by participants was 7.8. The prevalence of NMS in de novo patients in their study was even higher compared to that in our untreated subjects. Different measurements might be a reason, since they used simple yes/no questions, and we also used complicated scales. The high occurrence of NMS challenges the clinical management. Whether antiparkinsonian treatments are associated with NMS will directly influence the decision of pharmacologic therapies for PD.

Another interesting finding of this study is the apparent association between antiparkinsonian medications and the occurrence of NMS. In general, patients with antiparkinsonian treatments were more likely to be bad sleepers, depressed, and with constipation and cognition impairment compared with non-treated patients. When subgroups of dopaminergic medications were considered, patients taking levodopa plus agonists had worse sleep quality but less fatigue compared to levodopa alone treated. Moreover, constipation was significantly associated with LED but did not differ between patients with and without agonists. These findings partially confirmed previous studies. Barone et al. (2009) found that gastrointestinal, pain, urinary, sleep, skin disorder, and 'miscellaneous' (including olfactory dysfunction, dysgeusia, diplopia, weight change and sexual dysfunction) NMS were more frequent in treated subgroups.

There is still debate on whether dopaminergic medication can contribute to the development or deterioration of NMS such as poor nocturnal sleep, depression, constipation or cognitive impairment alone. Previous animal studies had proposed that higher dose of dopaminergic medication might inhibit sleep through action at post-synaptic D1 and possibly D2 receptors (Monti et al. 1989; Laloux et al. 2008). However, several open-label studies suggested that dopaminergic drugs could improve sleep dysfunction in PD (Eggert et al. 2008; Honig et al. 2009), and the RECOVER trial found that Rotigotine could improve nocturnal sleep disturbances and possibly dopaminergic nonmotor daytime symptoms (such as fatigue and mood) as well (Trenkwalder et al. 2011). To be noted, these agonists were not available in China when the study was carried out. Recent studies in animal models of PD suggested that L-dopa might interfere with norepinephrine (NE) and serotonin (5-HT) function in affect-related brain structures and induce symptoms of depression (Navailles et al. 2010a, b). A double blind randomized placebo-controlled trial also found that acute challenge with pramipexole had a negative effect on mood and fatigue (Drijgers et al. 2012). However, Chaudhuri and Schapira (2009) summarized several clinical trials on the effects of dopaminergic therapy on depression in PD, most of which suggested benefits from dopaminergic medications.

A possible constipating effect of dopaminergic treat- ment in PD is still debated, with controversial evidence. However, the potentiation of a pre-existing delay in colonic transit by dopamine has been presumed (Sakakibara et al. 2008). Besides, findings of studies on cognitive effect of levodopa and dopamine agonists are heterogeneous, and no clear conclusion can be drawn in relation to the effects of dopaminergic therapy on different cognitive domains. Previous study reported that dopaminergic medication could improve or impair cognitive performance depending on the nature of the task and the basal level of dopamine function in underlying cortico-striatal circuitry (Cools et al. 2001). Although the causality of NMS and dopaminergic therapy is not clear, since most of NMS in PD are likely multi-factorial in etiology, our findings suggest that dopaminergic medication needs to be considered in the management of NMS in the early stage of PD.

The pathology of PD suggested that Lewy bodies exist not only in the midbrain but also in the olfactory tract, anterior olfactory nucleus, and medulla oblongata, even up to the cortex (Braak et al. 2003). Various affected regions would result in different NMS, and it might be possible that several NMS coexist at the same time. Our study suggested when potential covariant were controlled, sleep quality, depression, and fatigue were significantly associated with each other. This phenomenon has been reported in previous studies. In patients with a major depression disorder, fatigue and sleep disturbances were common and had a high prevalence (Baldwin and Papakostas 2006). Our study indicated the coexistence of these three NMS, but coadministration of agonists with L-dopa improves fatigues while worsens sleep quality. In clinical practice, clinicians should pay attention to the other two NMS when one of them is presented and avoid medications, which might induce or aggravate symptoms.

Due to the limitation of the inclusion criteria at the baseline in the clinical trials, we excluded patients with MMSE $\leq 24$; therefore, our results only applied to the early PD patients without obvious symptoms of dementia. 20.8\% patients involved in this study did not complete all the evaluation forms, which might have caused a selection bias. We compared the demographic and clinical characteristics between patients who completed and did not complete all the evaluation scales. These characteristics were not different in the two groups, besides duration. Another limitation of our study was that the untreated patients had less disease burden compared to the treated patients, which might bias the results. Therefore, we used regression model to control potential influencing factors, which might have minimized the bias. Moreover, when we investigated the association between medication and NMS only in treated patients, there was still evidence that dopaminergic treatment was associated with NMS, no matter in subgroups or in LED. However, this is a cross-sectional study, which only provided evidence of the associations for clinical consideration. Prospective clinical observations would be needed to investigate the causality between NMS and dopaminergic 
therapies.

In conclusion, NMS are highly prevalent in our Chinese PD population, either related to antiparkinsonian treatment or coexisting with each other. Well recognition of NMS and their related factors would help in disease diagnosis and management.

\section{Acknowledgements}

The authors would like to thank all participants in this study, as well as all collaborators from the Chinese Parkinson Study Group (CPSG) for data collection used in this study. This work was supported by grants from the Ministry of Science and Technology of China (2006AA02A408 and 2012AA02A514) and the Beijing High Standard Health Human Resource Cultural Program in Health System (2009-1-12).

\section{Members of Chinese Parkinson Study Group (CPSG):}

Piu Chan (Xuanwu Hospital of Capital Medical University), Yanming Xu (West China Hospital of Sichuang University), Jun $\mathrm{Xu}$ (The Affiliated Gulou Hospital of Nanjing University), Yuming Xu (The First Affiliated Hospital of Zhengzhou University); Chunfeng Liu (The Second Affiliated Hospital of Suzhou University), Zhenyu Wang (The Third Affiliated Hospital of Henan University of Traditional Chinese Medicine), Ping Wang (The Inner Mongolian People's Hospital), Guohua Hu (The Second Affiliated Hospital of Jilin University), Weizhi Wang (The Second Affiliated Hospital of Harbin University), Baorong Zhang (The Second Affiliated Hospital of Zhejiang University), Zhanhua Liang (The First Affiliated Hospital of Dalian University), Anmu Xie (The Affiliated Hospital of Qindao University), Shengdi Chen (The Affiliated Ruijin Hospital of Shanghai Jiaotong University), Beisha Tang (Xiangya Hospital of Central Southern University), Benyan Luo (The First Affiliated Hospital of Zhejiang University), Wen Lü (The Affiliated Sir Sun Yat-Sen Hospital of Zhejiang University), Shenggang Sun (The Affiliated Union Hospital of Tongji Medical College, Huazhong University of Science \& Technology), Ming Shao (The First Affiliated Hospital of Guanzhou Medical College), Tao Feng (The Affiliated Tiantan Hospital of Capital Medical University), Zhuolin Liu (The First Hospital of Sun Yat-Sen University); Yiming Liu (Qilu Hospital of Shangdong University), Qinyong Ye (The Affiliated Union University of Fujian Medical University), Haibo Chen (Beijing Hospital), Xinhua Wan (Peking Union Medical College Hospital), Xia Sheng (The Affiliated Hospital of Xuzhou Medical College).

\section{Conflict of Interest}

The authors declare no conflict of interest.

\section{References}

Baldwin, D.S. \& Papakostas, G.I. (2006) Symptoms of fatigue and sleepiness in major depressive disorder. J. Clin. Psychiatry, 67 Suppl 6, 9-15.

Barone, P., Antonini, A., Colosimo, C., Marconi, R., Morgante, L., Avarello, T.P., Bottacchi, E., Cannas, A., Ceravolo, G., Ceravolo, R., Cicarelli, G., Gaglio, R.M., Giglia, R.M., Iemolo, F., Manfredi, M., et al. (2009) The PRIAMO study: A multicenter assessment of nonmotor symptoms and their impact on quality of life in Parkinson's disease. Mov. Disord., 24, 16411649.

Braak, H., Del Tredici, K., Rüb, U., de Vos, R.A., Jansen Steur, E.N. \& Braak, E. (2003) Staging of brain pathology related to sporadic Parkinson's disease. Neurobiol. Aging, 24, 197-211.

Chaudhuri, K.R. \& Schapira, A.H. (2009) Non-motor symptoms of Parkinson's disease: dopaminergic pathophysiology and treatment. Lancet Neurol., 8, 464-474.

Cools, R., Baker, R.A., Sahakian, B.J. \& Robbins, T.W. (2001) Enhanced or impaired cognitive function in Parkinson's disease as a function of dopaminergic medication and task demands. Cereb. Cortex, 11, 1136-1143.

Drijgers, R.L., Verhey, F.R., Tissingh, G., van Domburg, P.H., Aalten, P. \& Leentjens, A.F. (2012) The role of the dopaminergic system in mood, motivation and cognition in Parkinson's disease: a double blind randomized placebo-controlled experimental challenge with pramipexole and methylphenidate. J. Neurol. Sci., 320, 121-126.

Duncan, G.W., Khoo, T.K., Yarnall, A.J., O’Brien, J.T., Coleman, S.Y., Brooks, D.J., Barker, R.A. \& Burn, D.J. (2013) Healthrelated quality of life in early Parkinson's disease: the impact of nonmotor symptoms. Mov. Disord., [Epub ahead of print].

Eggert, K., Schrader, C., Hahn, M., Stamelou, M., Rüssmann, A., Dengler, R., Oertel, W. \& Odin, P. (2008) Continuous jejunal levodopa infusion in patients with advanced parkinson disease: practical aspects and outcome of motor and nonmotor complications. Clin. Neuropharmacol., 31, 151-166.

Honig, H., Antonini, A., Martinez-Martin, P., Forgacs, I., Faye, G.C., Fox, T., Fox, K., Mancini, F., Canesi, M., Odin, P. \& Chaudhuri, K.R. (2009) Intrajejunal levodopa infusion in Parkinson's disease: a pilot multicenter study of effects on nonmotor symptoms and quality of life. Mov. Disord., 24, 1468-1474.

Laloux, C., Derambure, P., Houdayer, E., Jacquesson, J.M., Bordet, R., Destée, A. \& Monaca, C. (2008) Effect of dopaminergic substances on sleep/wakefulness in saline- and MPTP-treated mice. J. Sleep Res., 17, 101-110.

Litvan, I., Bhatia, K.P., Burn, D.J., Goetz, C.G., Lang, A.E., McKeith, I., Quinn, N., Sethi, K.D., Shults, C. \& Wenning, G.K.; Movement Disorders Society Scientific Issues Committee. (2003) Movement Disorders Society Scientific Issues Committee report: SIC Task Force appraisal of clinical diagnostic criteria for Parkinsonian disorders. Mov. Disord., 18, 467-486.

Monti, J.M., Jantos, H. \& Fernández, M. (1989) Effects of the selective dopamine D-2 receptor agonist, quinpirole on sleep and wakefulness in the rat. Eur. J. Pharmacol., 169, 61-66.

Navailles, S., Benazzouz, A., Bioulac, B., Gross, C. \& De Deurwaerdère, P. (2010a) High-frequency stimulation of the subthalamic nucleus and L-3,4-dihydroxyphenylalanine inhibit in vivo serotonin release in the prefrontal cortex and hippocampus in a rat model of Parkinson's disease. J. Neurosci., 30, 2356-2364.

Navailles, S., Bioulac, B., Gross, C. \& De Deurwaerdère, P. (2010b) Serotonergic neurons mediate ectopic release of dopamine induced by L-DOPA in a rat model of Parkinson's disease. Neurobiol. Dis., 38, 136-143.

Politis, M., Piccini, P., Pavese, N., Koh, S.B. \& Brooks, D.J. (2008) Evidence of dopamine dysfunction in the hypothalamus of patients with Parkinson's disease: an in vivo $11 \mathrm{C}$-raclopride PET study. Exp. Neurol., 214, 112-116.

Sakakibara, R., Uchiyama, T., Yamanishi, T., Shirai, K. \& Hattori, T. (2008) Bladder and bowel dysfunction in Parkinson's disease. J. Neural Transm., 115, 443-460.

Tomlinson, C.L., Stowe, R., Patel, S., Rick, C., Gray, R. \& Clarke, C.E. (2010) Systematic review of levodopa dose equivalency reporting in Parkinson's disease. Mov. Disord., 25, 26492653.

Trenkwalder, C., Kies, B., Rudzinska, M., Fine, J., Nikl, J., Honczarenko, K., Dioszeghy, P., Hill, D., Anderson, T., Myllyla, V., Kassubek, J., Steiger, M., Zucconi, M., Tolosa, E., Poewe, W., et al. (2011) Rotigotine effects on early morning motor function and sleep in Parkinson's disease: a doubleblind, randomized, placebo-controlled study (RECOVER). Mov. Disord., 26, 90-99. 
Wolters, E.Ch. \& Braak, H. (2006) Parkinson's disease: premotor 70, 309-319. clinico-pathological correlations. J. Neural Transm. Suppl., 